Final Report: Biological and Synthetic Nanostructures Controlled at the Atomistic Level

A. Williamson, T. van Buuren

April 15, 2007 
This document was prepared as an account of work sponsored by an agency of the United States Government. Neither the United States Government nor the University of California nor any of their employees, makes any warranty, express or implied, or assumes any legal liability or responsibility for the accuracy, completeness, or usefulness of any information, apparatus, product, or process disclosed, or represents that its use would not infringe privately owned rights. Reference herein to any specific commercial product, process, or service by trade name, trademark, manufacturer, or otherwise, does not necessarily constitute or imply its endorsement, recommendation, or favoring by the United States Government or the University of California. The views and opinions of authors expressed herein do not necessarily state or reflect those of the United States Government or the University of California, and shall not be used for advertising or product endorsement purposes.

This work was performed under the auspices of the U.S. Department of Energy by University of California, Lawrence Livermore National Laboratory under Contract W-7405-Eng-48. 


\section{Final Report: \\ Biological and Synthetic Nanostructures \\ Controlled at the Atomistic Level \\ (03-SI-001)}

PI: Andrew Williamson, PAT

Co-PI: Tony van Buuren, CMS

\section{Funded Collaborators:}

Olgica Bakajin (CMS), Giancarlo Cicero (PAT), B. Dick (CMS), R. Friddle (CMS), G. Galli (PAT/UC Davis), D. Gerion (PAT), J. Lee (CMS), A. Noy (CMS), D. Prendergast (PAT), F. Reboredo (PAT), T. Willey (CMS), N. Zaitseva (PAT)

\section{Non-funded collaborators:}

A.P. Alivisatos (UC Berkeley), D. Aruguete (UC Berkeley), Fanqing Chen (LBNL and Comparative Cancer Center-UCSF), Neil Drummond (University of Cambridge, UK.), J. Grossman (UC Berkeley), J. Herberg (CMS), T. Huser (CMS/UC Davis), D. Krol (UC Davis), S. Lane (PAT), R. Leon (SEGRF student, LLNL), M. Marcus (LBL), R. Needs (University of Cambridge, UK), J.Y. Raty (Belgium), E. Schwegler (PAT), C. Talley (CMS).
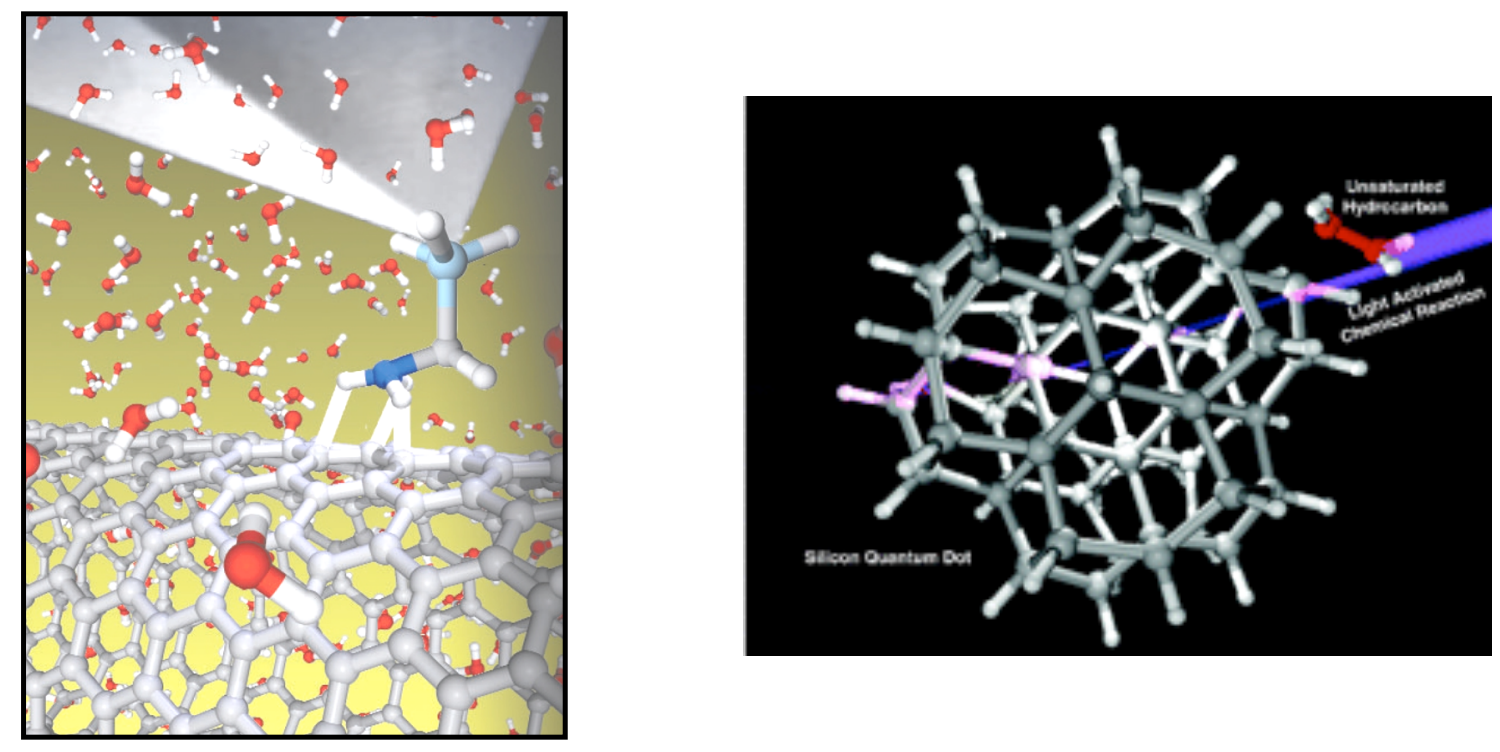


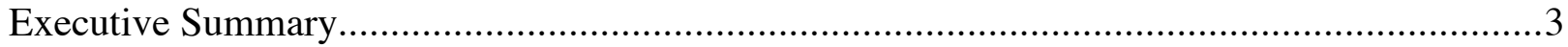

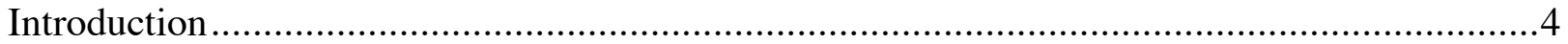

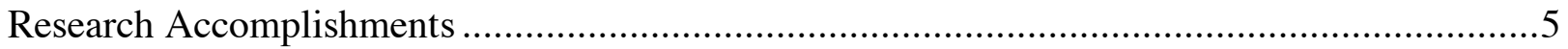

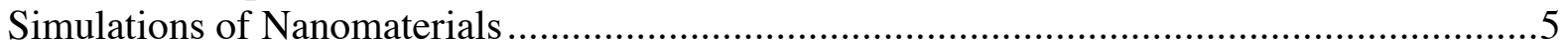

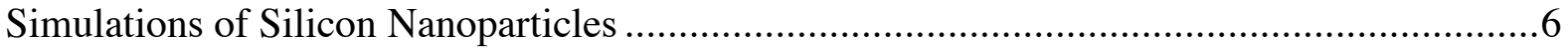

Modeling the Effect of Organic Functionalization of Silicon Quantum Dots .......................7

Simulations of Germanium Nanoparticles...........................................................

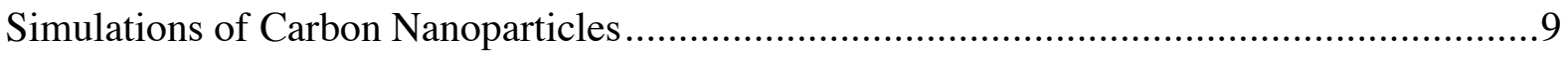

Modeling of the Opto-Electronic Properties of Nanodiamonds........................................9

Alloy nanoparticles and the search for new materials: SiC ........................................ 10

Simulations of the self-healing of CdSe quantum dots ................................................ 11

Modeling the Growth and Structure of CdSe Nanoparticles ........................................ 12

Simulations of the Effects of Solvation on Nanostructures ............................................ 12

Simulations of the structural and magnetic properties of Cobalt Nanoparticles...................15

Calculations of plasmons in metallic nanoparticles used for SERS ...............................15

Synthesis and Characterization of Silicon and Germanium Nanoparticles Using Physical

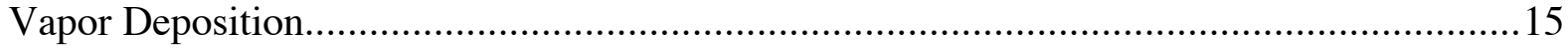

Chemical Synthesis and Characterization of Nanoparticles and Superlattices and Wires ......16

Synthesis and Applications of Functionalized CdSe Nanoparticles.................................17

Applications of carbon nanotubes as novel nanoscale stationary phases for molecular

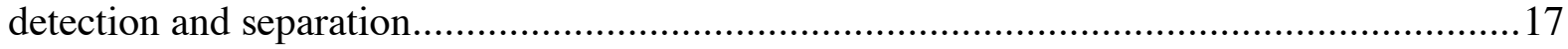

Chemical Force Microscopy Measurements separation ............................................. 18

Carbon Nanotube Device Fabrication and Characterization........................................... 18

Publications, Patents, Invited Talks and Other SI Related Activities ...................................20

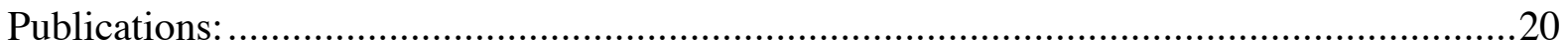

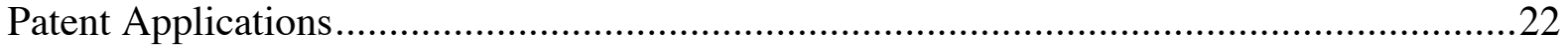

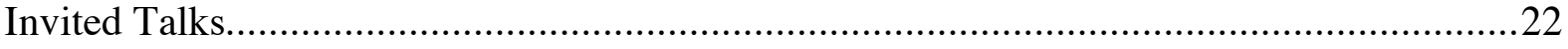

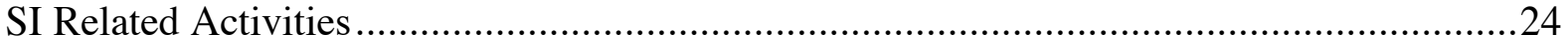


Final Report: 03-SI-001, PI: A.J. Williamson

\section{EXECUTIVE SUMMARY}

Nanotechnology holds great promise for many application fields, ranging from the semiconductor industry to medical research and national security. Novel, nanostructured materials are the fundamental building blocks upon which all these future nanotechnologies will be based. In this Strategic Initiative (SI) we conducted a combined theoretical and experimental investigation of the modeling, synthesis, characterization, and design techniques which are required to fabricate semiconducting and metallic nanostructures with enhanced properties. We focused on developing capabilities that have broad applicability to a wide range of materials and can be applied both to nanomaterials that are currently being developed for nanotechnology applications and also to new, yet to be discovered, nanomaterials.

During this 3 year SI project we have made excellent scientific progress in each of the components of this project. We have developed first-principles techniques for modeling the structural, electronic, optical, and transport properties of materials at the nanoscale. For the first time, we have simulated nanomaterials both in vacuum and in aqueous solution. These simulation capabilities harness the worldleading computational resources available at LLNL to model, at the quantum mechanical level, systems containing hundreds of atoms and thousands of electrons. Significant advances in the density functional and quantum Monte Carlo techniques employed in this project were developed to enable these techniques to scale up to simulating realistic size nanostructured materials. We have developed the first successful techniques for chemically synthesizing crystalline silicon and germanium nanoparticles and nanowires. We grew the first macroscopic, faceted superlattice crystals from these nanoparticles. We have also advanced our capabilities to synthesize semiconductor nanoparticles using physical vapor deposition techniques so that we are now able to control of the size, shape and surface structure of these nanoparticles. We have made advances in characterizing the surface of nanoparticles using x-ray absorption experiments.

Throughout this SI a number of long-term, strategic external collaborations have been established. These collaborations have resulted in 30 joint publications, strategic hires of postdocs and graduate students from these groups into groups at LLNL and the submission of joint research grants. We have developed collaborations on the theory and modeling of nanomaterials with the groups of Profs. Ceder and Marzari (MIT), Crespi (Penn State), Freeman (Northwestern), Grossman and Lester (UC Berkeley), Mitas (North Carolina State), and Needs (Cambridge). We are collaborating with Dr. Alivisatos's group in the Molecular Foundry at Lawrence Berkeley Laboratory on the fabrication, characterization and modeling of inorganic nanomaterials. We are working with Prof. Majumdar's group at UC Berkeley on the characterization of nanomaterials. We are working with the molecular diamond group at ChevronTexaco who has developed a process for extracting mono-disperse samples of nano-scale diamonds from crude oil. We are collaborating with Dr. Chen at UCSF to develop CdSe nanoparticle-biolabels.

As a result of the outstanding scientific achievements and the long-term collaborations developed during this strategic initiative we have been extremely successful in obtaining external funding to continue and grow this research activity at the LLNL. We have received two DARPA grants to support the further development of our computational modeling techniques and to develop carbon nanotube based molecular separation devices. We have received two new Office of Science BES grants to support our nanomaterials modeling and synthesis projects. We have received funding from the NA22 office of DOE to develop the materials modeling capabilities begun in this SI for modeling detector materials. We have received funding from Intel Corporation to apply the modeling techniques developed in this initiative to examine silicon nanowires fabricated on computer chips. We are also pursuing several additional sources of funding from BES, the DHS, and NIH to support the continuation of the research programs developed in this SI.

The remainder of this report and the attached publications describe the background to this SI research project and the details of the scientific achievements that have been made. 
Final Report: 03-SI-001, PI: A.J. Williamson

\section{INTRODUCTION}

In this SI project we chose to focus on specific, high profile nanoscience problems with key connections to technologies of interest to new LLNL Laboratory programs (e.g. labeling and sensing devices). In particular, we focused on the science underlying the design, at the microscopic scale, of nanostructures and nanostructured materials for the detection of chemical and biological markers. To build the nanoscience competency needed for a successful development of nanotechnology in the field of chemical tags and biological sensors, we conducted the following research activities:

- Develop specific and predictive ab-initio simulation techniques to model organic/inorganic interfaces between nanoparticles and carbon nanotubes tubes and organic markers. Use the results of these simulations to guide and complement characterization and functionalization experiments and to provide guidance to synthesis experiments searching for new materials which are biocompatible and have desired, targeted properties.

- Develop experimental capabilities to produce size-selected, non-toxic, nanoparticles with controllable stoichiometry, using chemical synthesis and physical vapor deposition techniques. Compare systematically the physical and chemical properties of nanostructures obtained with different preparation methods, as well as the results of simulations.

- Develop spectroscopic capabilities to characterize the structural and optical properties of nanoparticles, with special emphasis towards the study of individual particles and the interface between inorganic nanomaterials and organic molecules, in vacuum and solution.

- Develop design guidelines for carbon nanotube elements that will increase their collection specificity using advanced AFM techniques coupled with state-of-the-art ab-initio simulations.

We accomplished the goals outlined above by taking advantage of the unique capabilities and expertise present at LLNL:

- LLNL has unique computational expertise in performing ab initio density functional and Quantum Monte Carlo calculations for real materials (members of QSG group in PAT). In addition, the LLNL teraflop scale ASC computing facilities are second to none. In these fields, we bring a combined set of relevant computer codes, computational power and expertise that is unrivaled both nationally and internationally.

- We have developed world class capabilities for synthesizing nanoparticles using Physical Vapor Deposition (Tony van Buuren) and chemical synthesis (Daniele Gerion and Natalia Zaitseva).

- We are well positioned to carry out spectroscopic measurements on quantum dots using x-ray absorption and emission spectroscopy (T. van Buuren and collaborators at the ALS). The comparison of experimental results with those of computer simulations provides a comprehensive understanding of the physical and chemical properties of surface and interface properties. Successful comparisons have already been carried out for $\mathrm{Si}, \mathrm{Ge}$ and $\mathrm{CdSe}$ nanoparticles

- We have unique experimental and characterization capabilities for carbon nanotube arrays (A. Noy and O. Bakajin). 
Final Report: 03-SI-001, PI: A.J. Williamson

\section{RESEARCH ACCOMPLISHMENTS}

\section{Simulations of Nanomaterials}

A substantial part of this project was devoted to simulating the structural, electronic, optical, and transport properties of group IV semiconductor nanostructures such as quantum dots and nanotubes interfaced with specific biological and chemical media, in solution.

An essential prerequisite to understanding how to tailor group IV nanostructure interfaces with desired organic media is a thorough study of the influence of surface structure and passivation on the ground state properties of semiconductor quantum dots and carbon nanotubes; this study needed to be first carried out in vacuum and then in aqueous solution. In particular, in order to tailor the properties of quantum dots for specific sensing applications, it is important (i) to separate the effects of quantum confinement from those due to the surface, (ii) to unravel the mechanisms by which preparation conditions can influence the dot atomistic structure, and thus its optical properties, and (iii) to understand whether different preparation techniques can yield nanoparticles with substantially different physical properties. To address these issues, we used computational tools to investigate semiconductor nanostructures in vacuum and in solution.

In our investigations, we used ab-initio molecular dynamics (MD) techniques to predict the structural and electronic properties of semiconductor dots interfaced with desired organic media and surrounded by a solvent. Furthermore, to accurately treat the effects of exchange and correlation, we used Quantum Monte Carlo (QMC) techniques to compute optical gaps. These are highly accurate computational techniques, which do not require fitting any experimental data, and which can provide results to complement and interpret experiments. Both techniques provide a full quantum mechanical treatment of interatomic interactions, but make use of different approximations for the exchange and correlation potential.

We note that while several empirical potentials exist to describe some conventional surfaces, which have been derived from fitting solid-state data, appropriate potentials for nanoparticles are not available. The ab initio molecular dynamics methodologies and the expertise developed in the Quantum Simulation group at LLNL as part of this SI are powerful tools for investigating the properties of nanostructures in a full quantum mechanical manner, with no fit to experiment, and with predictive capabilities. In contrast to traditional electronic structure approaches, temperature and disorder effects have been incorporated naturally in these calculations. Having predictive computational capabilities for quantum dots is essential since key data about these systems is difficult to extract from experiment. For example, while optical gaps and other electronic properties of semiconductor nanoclusters can be measured accurately, their dependence on the structure of the particle, in particular the surface termination, are difficult to determine precisely from experiment.

Both $a b$ initio MD techniques and QMC methods are computationally very expensive. However, using the institutional computing resources at LLNL, we have been able to apply abinitio MD techniques to nanoclusters of up to $5 \mathrm{~nm}$ size, and thus carry out calculations for dots and tubes which are directly comparable with experiment and are relevant for technological applications. The central code for our ab initio simulations was the QBox code developed at LLNL by Francois Gygi and Erik Draeger. Qbox is an implementation of large-scale ab initio molecular dynamics. In 2006 it was awarded the Gordon Bell prize at the 2006 
Supercomputing conference for performing ab-initio simulations to achieve a record breaking 207 TFlops performance. The central code used for the QMC simulations was a localized orbital based code developed at LLNL by A.J. Williamson, R.Q. Hood and J.C. Grossman, which can treat both periodic and isolated systems.

\section{Simulations of Silicon Nanoparticles}

We used use ab-initio molecular dynamics simulations to investigate the effects of surface reconstruction and the effect of different molecules to passivate the surface of nanodiamond, and silicon and germanium nanoclusters. We used a combination of Density Functional Theory (DFT) and highly accurate, quantum Monte Carlo calculations to predict the relative stabilities of hydrogenated silicon nanoparticles (up to $2 \mathrm{~nm}$; see Fig.1) with reconstructed and unreconstructed surfaces. We identified previously unconsidered reconstructions which are unique to the highly curved
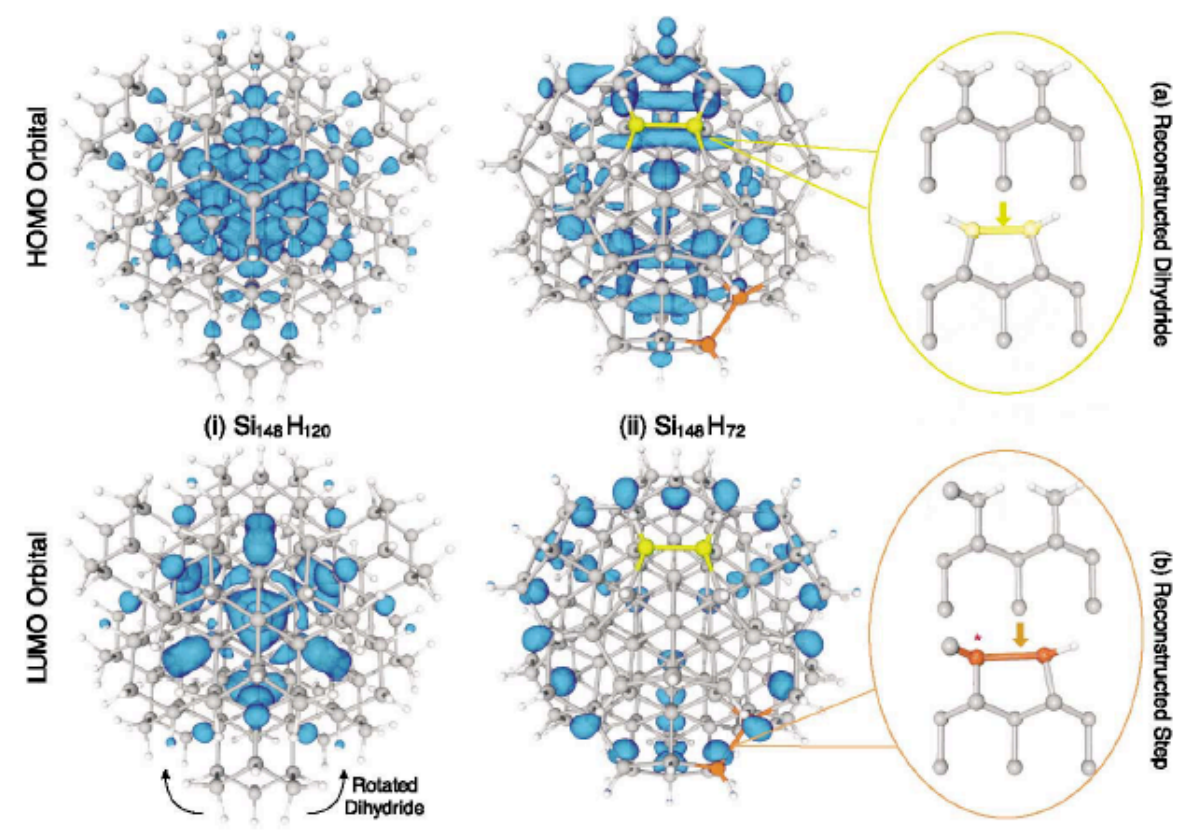

Fig. 1: Unreconstructed (left hand side) and reconstructed (right hand side) hydrogen passivated Si clusters (1.8 $\mathrm{nm}$ in size). Charge density isosurfaces represent $50 \%$ of the peak amplitude. (from A.Puzder, A.Williamson, F.Reboredo and G.Galli, Phys. Rev.Lett. 91, 157405-1 (2003)).

surfaces of nanostructured materials. We predicted that reconstructions of surface steps dramatically reduce the optical gap and decrease excitonic lifetimes by localizing the band edge electronic states on the surface of the clusters. These predictions provide an explanation of both the variations in the photoluminescence spectra of colloidally synthesized nanoparticles and observed deep gap levels in porous silicon. In addition, our predictions highlight microscopic features of nanoparticle surfaces which affect their interactions with external media such as solvents and organic molecules used to functionalize the particles. We also investigated the effect of preparation conditions on the properties of Si nanoparticles and, in particular, we investigated the effect of the presence of oxygen during synthesis (see Fig.2). 


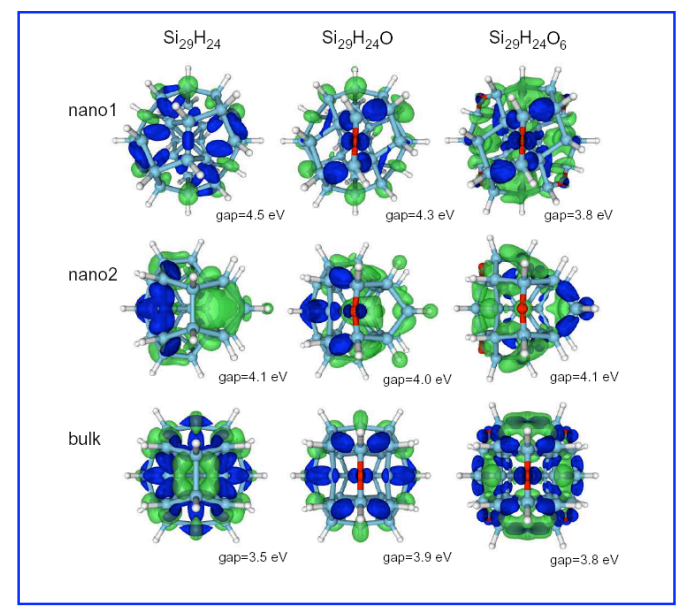

Fig.2: The effect of oxygen on the highest occupied molecular orbital (blue) and on the lowest unoccupied molecular orbital (green) of three reconstructions of $1 \mathrm{~nm}$ hydrogenated Si clusters (nano1 and nano2, obtained from direct ab-initio MD simulations mimicking a high temperature synthesis process and bulk, obtained from a fragment of crystalline bulk Si). [from E.Draeger, J.Grossman, A.Williamson, and G.Galli, J.Chem.Phys. 2004]

We also investigated photo-induced attachments of organic molecules to silicon dots. In particular, we studied the influence of optical activation on functionalization reactions of $\mathrm{Si}$ quantum dots with unsaturated hydrocarbons. We found that the energy barrier for the replacement of silicon-hydrogen with silicon carbon bonds is dramatically reduced if the Si dot is optically excited (see Fig. 3). Our results have provided an explanation for recent experiments on optically excited porous Si. In addition our calculations point at the existence of an intermediate spin polarized state formed by the dot and an alkene or alkyne, upon relaxation after absorbing a photon. This state could be detected experimentally, by e.g. electron spin resonance measurements. Based on the results of our calculations as a function of the dot size, varied from 0.8 to $1.5 \mathrm{~nm}$, we have proposed that light activated reactions could be used to functionalize and size select silicon quantum dots at the same time.

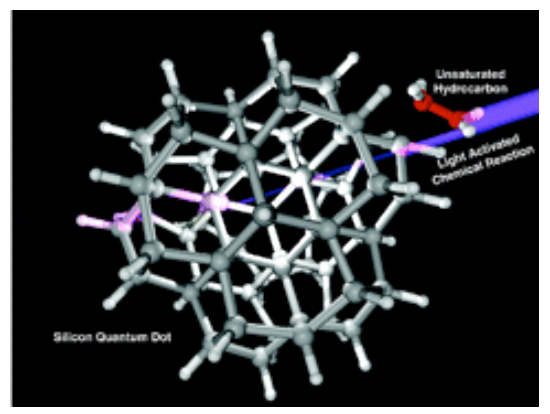

Fig.3: Schematic representation of hydrosylation reaction studied theoretically as the simplest step to functionalization of Si dots. [from F.Reboredo, E.Schwegler, G.Galli, J. Amer. Chem.Soc. 125, 15243 (2003).].

\section{Modeling the Effect of Organic Functionalization of Silicon Quantum Dots}

We also performed a theoretical study of silicon nanoparticles functionalized with alkyl chains whose length varies from 1 to 4 carbon atoms. An example of an alkyl terminated nanoparticle is shown in Fig. 4. These simulations revealed several interesting changes in the optical properties of silicon quantum dots as a function of surface passivation. In particular, we have compared hydrogen passivated dots with those having alkyl groups at the surface. We found 
that, while on clusters with reconstructed surfaces a complete alkyl passivation is possible, steric repulsion prevents full passivation of Si dots with unreconstructed surfaces. In addition,

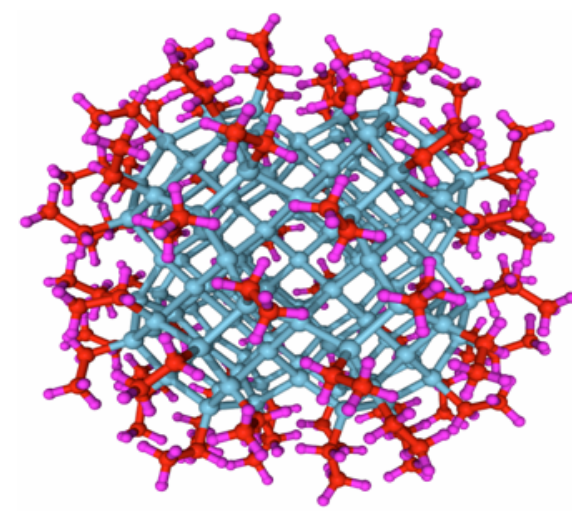

Fig. 4 Simulation of an alkyl terminated silicon nanoparticle. our calculations showed that steric repulsion may have a dominant effect in determining the surface structure, and eventually the stability of alkyl passivated clusters, with results dependent on the length of the carbon chain. Alkyl passivation weakly affects optical gaps of silicon quantum dots, while it substantially decreases ionization potentials and electron affinities and affects their excited state properties. On the basis of these simulations we proposed a novel technique by which alkyl terminated quantum dots can be size selected by taking advantage of the change in ionization potential as a function of the cluster size.

\section{Simulations of Germanium Nanoparticles}

We conducted a computational investigation in close connection with experiment (A.Van Buuren and his team) which has led to a joint publication on the use of the electronic density of states of nanoparticles to infer information about their electronic structure. An example of the detailed comparison between theory and experiment which we were able to obtain is shown in Fig.5.
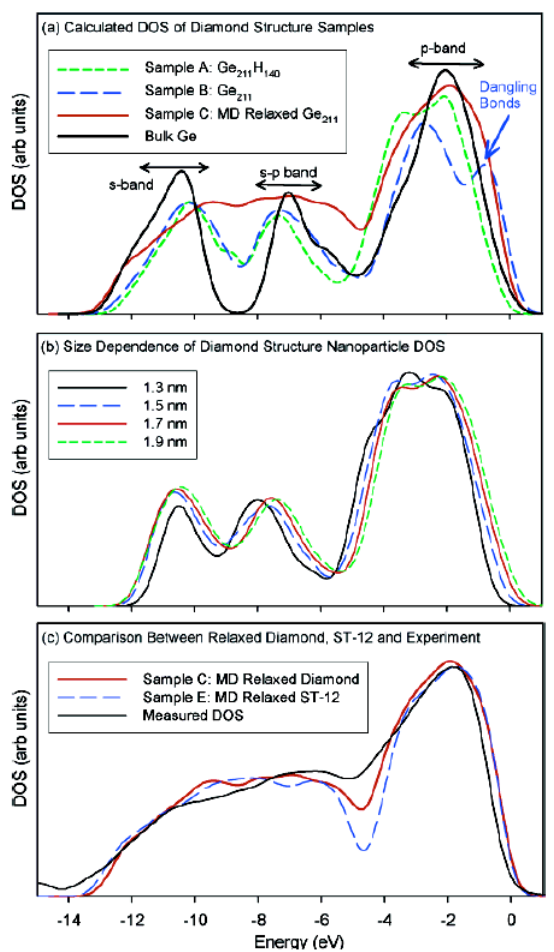

Fig.5: Predicted electronic Electronic density of states (DOS) of Ge clusters (a), calculated size dependance (b) and comparison with measurements performed at the ALS (c). [fromA.Williamson, C.Bodedt, A.Van Buuren. T.M.Willey, L.Terminello and G.Galli, Nanoletters 2004]. 


\section{Simulations of Carbon Nanoparticles}

In addition to $\mathrm{Si}$ and $\mathrm{Ge}$, we studied a variety of carbon nanoparticles. We investigated nanoscale carbon particles with a diamond-structure core and a surface reconstruction - a structure which we named "Bucky-Diamond". After the discovery of bucky diamond, we continued our investigation of assemblies of diamond nanoparticles with a fullerene core. Nanometer sized diamond has been found in meteorites and interstellar dusts, as well as in residues of detonation products and in ultra disperse (UDD) and ultra crystalline diamond (UCN) films. Remarkably, the size distribution of diamond nanoparticles appears to be peaked around $2-5 \mathrm{~nm}$, and to be largely independent of preparation conditions. We carried out an $a b$ initio theoretical study of the stability of nanodiamond as a function of surface hydrogen coverage and as a function of size (see Fig.6). We have found that at about $3 \mathrm{~nm}$, and for a broad range of $\mathrm{P}$ and $\mathrm{T}$ conditions, particles with bare, reconstructed surfaces become thermodynamically more stable than those with hydrogenated surfaces, thus preventing the formation of larger grains. However there exist thermodynamic conditions which can favor the formation of bulk flat surfaces and we suggest those may be attained in CVD growth. Our findings provide an explanation of the size distribution of nanodiamond found in meteorites and other extraterrestrial media, and in UCN and UDD films. Our results also provide an atomistic structural model of these films, based on the topology and structure of 2-3 nm bucky diamonds , and this is an important prerequisite in devising applications of UCN and UDD systems as NEMS and substrates for the attachment of biological molecules.
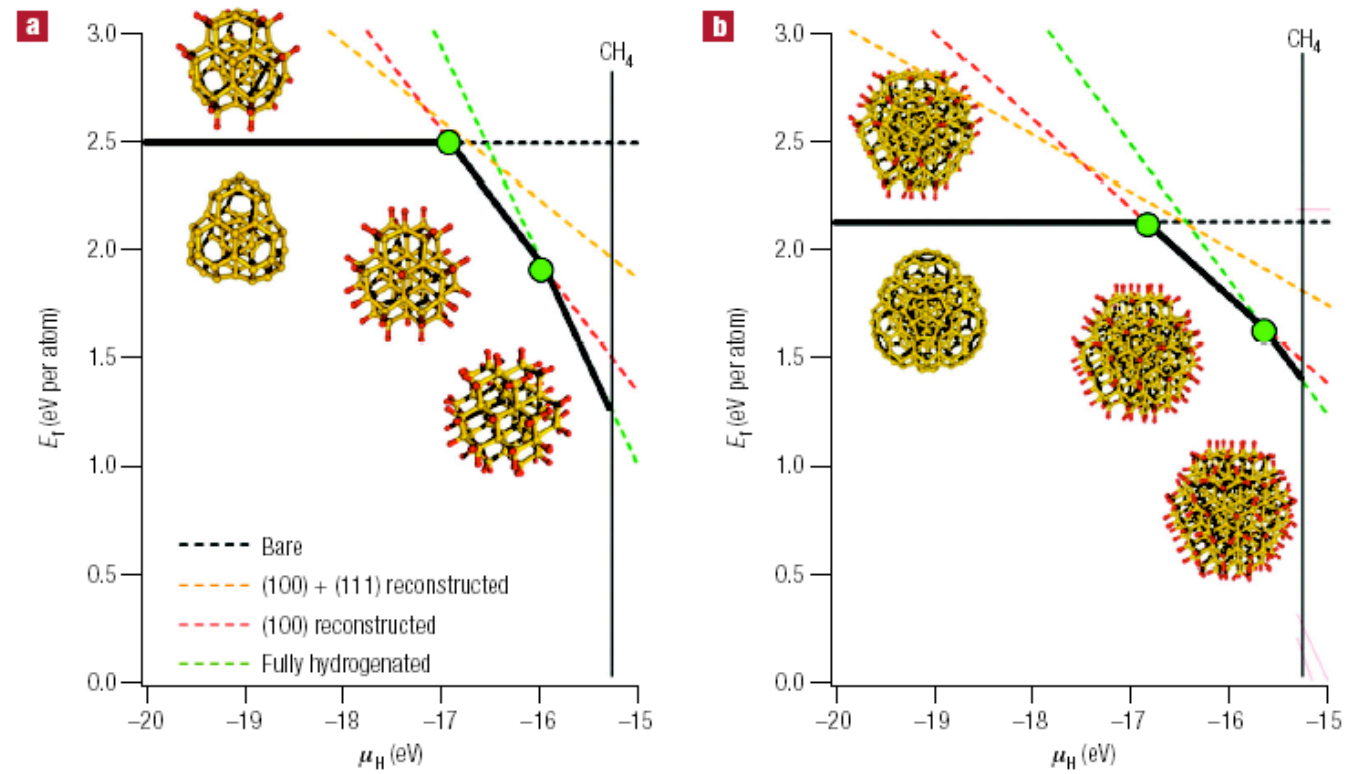

Fig.6: Formation energy of nanodiamonds as a function of hydrogen content, for two different cluster sizes (left and right, respectively).[from J.-Y. Raty and, G.Galli, Nature Materials 2003).].

\section{Modeling of the Opto-Electronic Properties of Nanodiamonds}


In addition to Bucky-Diamond we also study the properties of a class of carbon nanomaterials called diamondoids. These materials are a recently discovered nanoscale form of diamond that is attracting considerable scientific and technological interest. Despite repeated attempts over the last century, these molecules have evaded laboratory synthesis and only recently, when they were purified from petroleum sources, have they become available for technological applications and scientific investigation. We have developed a close collaboration with the group at Chevron who are producing these diamondoids (see Fig.7).

We have characterized the electronic structure of the band edge states of nanodiamonds. Using $\mathrm{x}$-ray absorption we performed the first measurements of the size dependence of the conduction

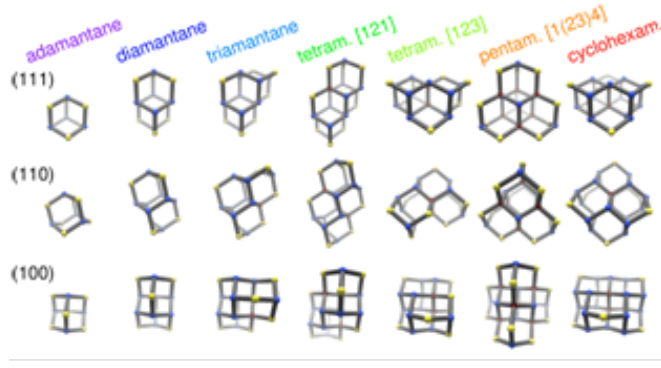

Figure 7: The structures of diamondoids band states in these materials. Our experiments, performed on diamondoids in the gas-phase, enable the investigation of perfectly mono-disperse, noninteracting clusters and showed many unexpected and exciting results. First, the band structure of bulk diamond, namely the " 2 nd band-gap" of diamond, usually associated with long-range crystallinity and order, appears and evolves in this series of clusters that are less than $1 \mathrm{~nm}$ in diameter. However, the core-exciton, evident in bulk diamond and another measure of long range order/crystallinity, does not

appear as expected in the diamondoids. Second, the lowest unoccupied states are overwhelmingly dominated by molecular orbitals associated with the hydrogen surface termination, and thus no band-edge shifts are evident, contrary to expectations from quantum confinement and our previous results for silicon and germanium nanoparticles.

In collaboration with Neil Drummond and Richard Needs (University of Cambridge) we have used Density Functional and Quantum Monte Carlo calculations to theoretically predict the size dependence of the band edge electronic states and optical gap of hydrogen terminated, carbon nanodiamonds. These calculations are the first quantitative predictions of the opto-electronic properties of nanodiamonds. They were made possible by the use of the linear scaling Quantum Monte Carlo techniques developed in this SI and long runs on the Thunder and MCR supercomputers. These calculations predicted a novel size dependence of the unoccupied electronic states, in which almost all the quantum confinement takes place in the valence band, while the conduction band energy is insensitive to nanoparticle size due to its delocalize nature.

\section{Alloy nanoparticles and the search for new materials: SiC}

We have carried out calculations of the optical and stability properties of $\mathrm{SiC}$ nanoparticles, and we made predictions of new nanostructured $\mathrm{SiC}$ materials emitting in the UV. In particular, we proposed that the stability and optical gaps of $\mathrm{SiC}$ dots can be engineered as a function of their size and surface structure and composition, so as to build materials for semiconductor based Ultra-Violet (UV) light sources. Our investigation was the first theoretical study of SiC nanoparticles and has addressed in detail two issues: the dependence of optical properties on size and surface composition and structure, and the stability of SiC dots as a function of surface termination. Our results allowed us not only to propose new nanostructured materials for UV 
light sources and new nanostructures for biological tags, but they also permitted the interpretation of recent, controversial high-pressure synthesis experiments where both $\mathrm{SiC}$ and Si dots may have been formed at the same time.

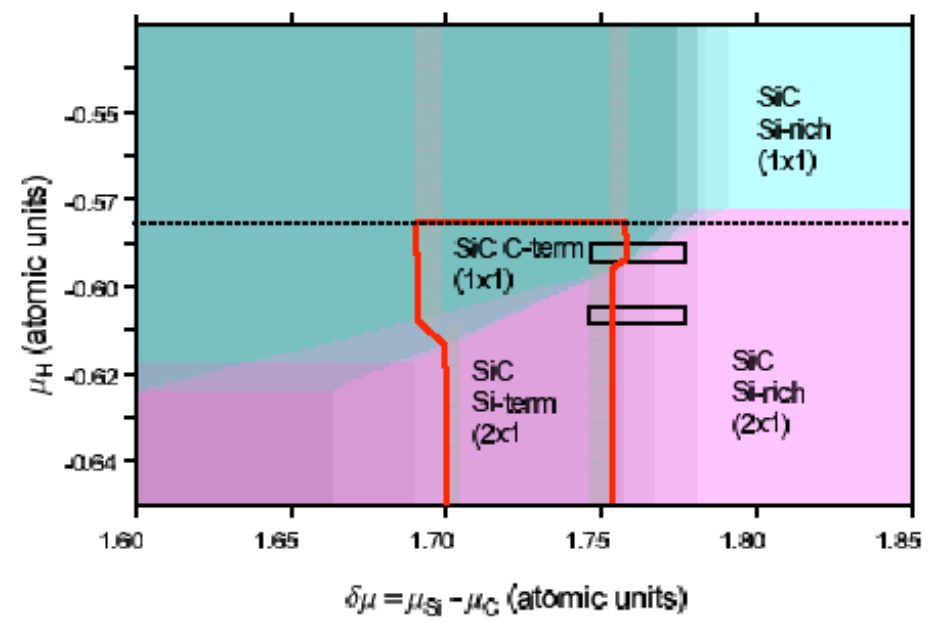

Fig.8: Stability of SiC quantum dots as a function of the $H$ chemical potential (vertical axis) and the difference between the $S i$ and $C$ chemical potentials (horizontal axis). The region marked in red denotes of chemical potentials for which SiC dots are thermodynamically stable. These are dots with either (1x1) C-terminated surfaces or (2x1) Si-terminated surfaces. The black rectangles denote values of chemical potentials under which both SiC and Si dots could be formed. [From F. Reboredo, L. Pizzagalli and G. Galli, Nanoletters 2004].

\section{Simulations of the self-healing of CdSe quantum dots}

The ability to routinely synthesize CdSe semiconductor nanocrystals with narrow size distributions has made CdSe nanoparticles one of the most promising building blocks for a new generation of nanoscale materials. The reproducibility of their optical absorption and emission properties has contributed to the popularity of CdSe nanocrystals for potential applications such as biological and medical sensors, displays, and quantum dot lasers. In spite of a wealth of experimental data reported for CdSe nanocrystals over the last decade, many fundamental questions regarding their structural and opto-electronic properties remained unanswered. In particular, although transmission electron microscopy (TEM) images usually indicate that $\mathrm{CdSe}$ nanoparticles adopt the wurtzite structure, it has been difficult to resolve their surface geometry and to assess the influence of organic ligands on surface reconstructions. Surface termination is known to affect the optical properties of Type IV and III-V semiconductors; in contrast the size dependence of the optical gap of CdSe nanoparticles is more robust and largely independent of the specific surface termination. The origin of this insensitivity of the gap to its environment is not well understood. Therefore, we carried out a series of ab-initio calculations to make direct contact with these experimental results. We performed simulations of the structural, electronic, and optical properties of CdSe nanoparticles up to $\sim 1.5 \mathrm{~nm}$ in size. The atomic structures of the clusters were relaxed both in vacuum and in the presence of surfactant ligands. In both cases, we predict significant geometrical rearrangements of the nanoparticle surface while the wurtzite core is maintained. These reconstructions lead to the opening of an optical gap without the aid of passivating ligands, thus "self-healing" the surface electronic 
structure. Our calculations also predicted the existence of a mid-gap state responsible for recently observed sub-band emission. Pictures of the simulated clusters and of some of their electronic states are given in Fig. 9.

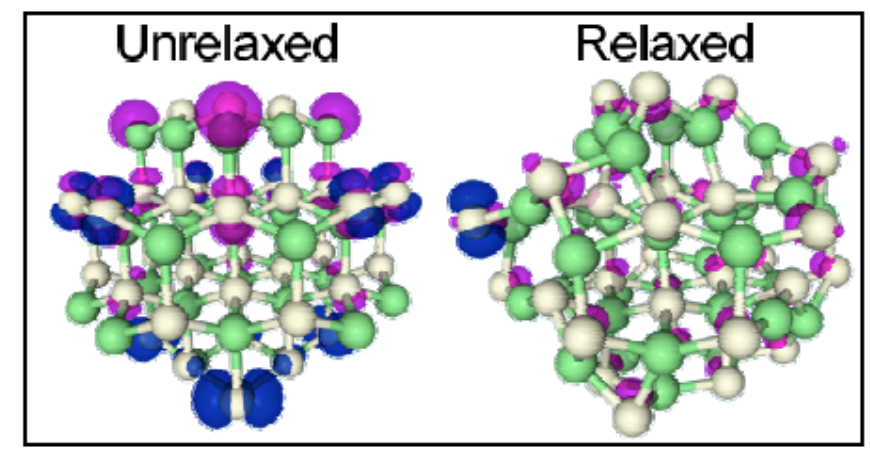

Fig. 9: Probability density of the highest occupied molecular orbital (HOMO) and lowest unoccupied molecular orbital (LUMO) for wurtzite and relaxed Cd33Se33 clusters. The blue (magenta) isosurface includes 50\% (25\%) of the HOMO (LUMO) density. The Cd atoms are green and the Se atoms are yellow in the ball and stick model. [from A.Puzder, A.Williamson. F.Gygi and G.Galli, Phys.Rev.Lett. 2004].

\section{Modeling the Growth and Structure of CdSe Nanoparticles}

We used Density Functional Theory calculations to study the structural, electronic and growth properties of CdSe quantum dots and rods. In collaboration with Prof. Alivisatos (LBNL) we studied the relative binding energies of different surfactants to surface facets of the CdSe dots. These calculations predicted a stronger binding of phosphonic acid surfactants to the (01-10) and (011-20) surfaces which make up the sides of CdSe nanorods than to the (0001) surfaces which make up the ends. This supports the hypothesis that the aspect ratio of CdSe nanorods is controlled by the presence of phosphonic acid molecules in the growth solution. Synthesis experiments performed at UCB support this hypothesis and show that the aspect ratio of nanorods can be controlled by altering the relative concentration of phosphonic acid and phosphine oxide molecules in the growth solution.

We collaborated with Deborah Aruguete (UCB) and Matthew Marcus (LBNL) to compare the structures of CdSe nanorods measured by EXAFS at the ALS with the predictions of quantum simulations. We have calculated the relaxed atomic geometry of CdSe nanorods with different diameters using Density Functional calculations. We then used a multiple scattering simulation of the X-ray absorption to predict the Extended X-ray Absorption Fine Structure (EXAFS) absorption spectra for the CdSe nanorods. By comparing our predicted EXAFS spectra with those measured at the ALS we have developed models for how the structure of CdSe nanorods evolves with their diameter. This recently developed capability to connect theoretically predicted atomistic structures with EXAFS measurements provides a new a valuable tool for understanding the properties of nanostructures.

\section{Simulations of the Effects of Solvation on Nanostructures}

The SI team has studied water at ambient conditions and in particular we have analyzed all the approximations of the theory and the techniques used. We have obtained novel and unexpected results which have been published in two papers in J. Chem. Phys. (J. Grossman et al., JCP 2004 and M. Allesch et al.. JCP 2004) which have had a notable impact in the field of water 
modeling and in that of Car-Parrinello simulations of hydrogen bonded systems. In particular, we carried out a series of Car-Parinello (CP) molecular dynamics simulations, aimed at assessing the accuracy of density functional theory in describing the structural and dynamical properties of water under ambient conditions. We found negligible differences in structural properties obtained using the Perdew-Burke-Ernzerhof or the Becke-Lee-Yang-Parr exchange and correlation energy functionals; size effects, although not fully negligible when using 32 molecule cells, were found to be rather small. In addition, we identified a wide range of values of the fictitious electronic mass $(\mu)$ entering the CP Lagrangian for which the electronic ground state is accurately described, yielding trajectories and average properties which are independent of the value chosen. However, care must be exercised not to carry out simulations outside this range, where structural properties may artificially depend on $\mu$ (see Fig. 10). In the case of an accurate description of the electronic ground state, and in the absence of proton quantum effects, we obtained an oxygen-oxygen correlation function which is over-structured compared to experiment.

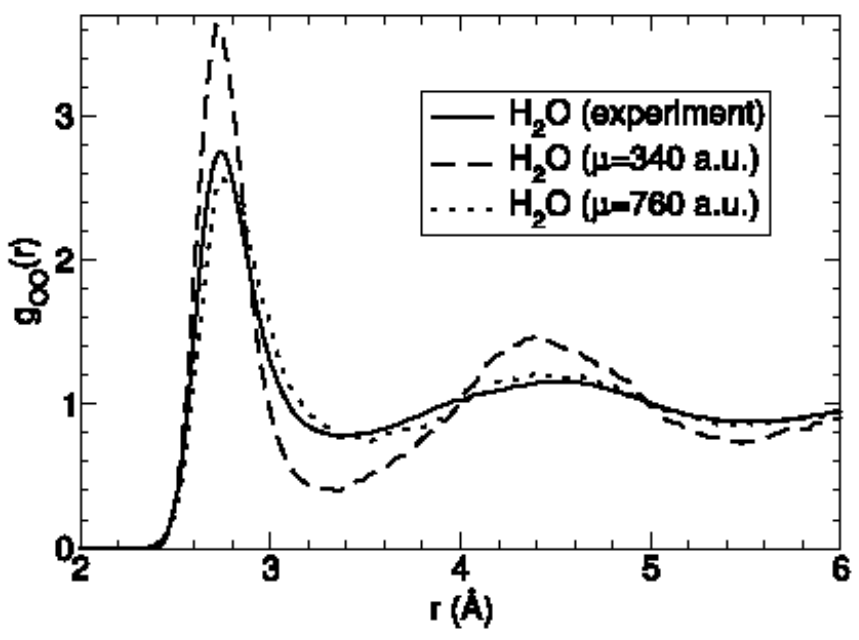

Fig. 10: Comparison of calculated oxygen-oxygen radial distribution functions with neutron diffraction experiments. The calculations shown are for the 32-H\$_2\$Owater molecule cells, the BLYP functional, and different values of the parameter mu entering the Car-Parrinello Lagrangian. (from J.Grossman, E.Schwegler, E.Draeger, F.Gygi and G.Galli, J.Chem.Phys. 2004).

We also performed a study of the structure of hydrophobic hydration in the case of small molecules, in particular silane and methane. We carried out a combination of classical and firstprinciples molecular dynamics simulations. Our results show that water molecules surrounding two small hydrophobic solutes are oriented in a similar fashion, and that the driving force for this orientation is the water-water interaction rather than the water-solute interaction. In contrast, the spatial distribution of water around the hydrophobic solute is strongly influenced by the solute, and the driving force for the observed distribution is largely a steric effect (see fig. 12). In addition to the size and structure of the solute, we find that the spatial distribution of water is sensitive to pressure. Using quantum simulations as a benchmark for classical potentials, we evaluated the accuracy of several empirical based modes in predicting detailed information regarding the structure of water around small hydrophobic solutes. Our results demonstrate that the radial and spatial distribution of water molecules around different solutes 
obtained classically and quantum mechanically agree rather well, indicating that classical potentials are well suited for examining these properties related to hydrophobic hydration structure. However, we do find that properties such as the angular distribution of water and hydrogen bond ring statistics (see Fig. 11) agree to a lesser extent and depend strongly on the classical potential employed.

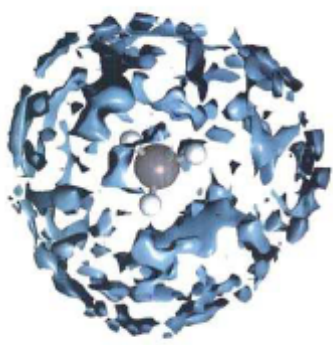

(a) methane

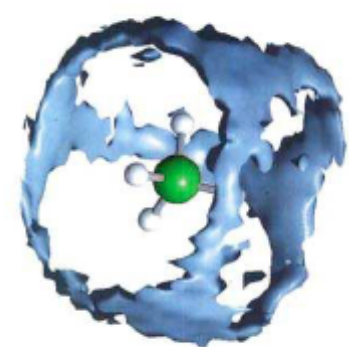

(b) silane

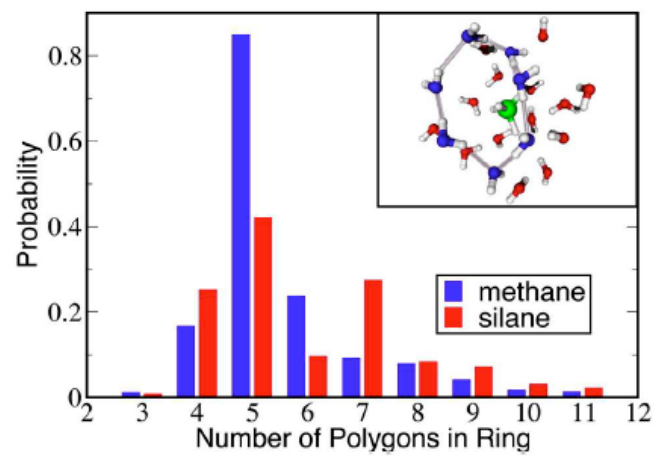

Fig.11 : In the upper part we show spatial distribution functions for water-methane and water-silane simulations. In each case, the same threshold is plotted for the local density of oxygen averaged over symmetric orientations of the solute. In the lower part of the picture we show the ring statistics obtained for silane and methane in first principles simulations. (from J.Grossman, E.Schwegler, G.Galli, submitted 2004).

We also investigated the impact of water on the optical absorption of prototypical silicon clusters with oxygen passivation. We approached this complex problem by assessing the contributions of three factors: chemical reactivity; thermal equilibration and dielectric screening. We found that the silanone $(\mathrm{Si}=\mathrm{O})$ functional group is not chemically stable in the presence of water and exclude this as a source of significant red shift in absorption in aqueous environments. We performed first principles molecular dynamics simulations of the solvation of an oxygenated silicon cluster with explicit water molecules at 300 K. We find a systematic 0.7 eV red shift in the absorption gap of this cluster, which we attribute to thermal strain of the molecular structure. Surprisingly, we find no observable screening impact of the solvent, in contrast with consistent blue shifts observed for similarly sized organic molecules in polar solvents. The predicted red shift is expected to be significantly smaller for larger Si quantum 
dots produced experimentally, guaranteeing that their vacuum optical properties are preserved even in aqueous environments. An example of the water/cluster sample and of the electronic states considered in our simulations is presented in Fig. 11.

\section{Simulations of the structural and magnetic properties of Cobalt Nanoparticles}

We also simulated the structural properties of metallic nanoparticles and in particular the properties of cobalt nanoparticles. Using density functional calculations we uncovered changes in the structural and magnetic properties of pristine cobalt clusters upon $\mathrm{CO}$ chemisorption. Our results showed that the binding energies of $\mathrm{CO}$ to $13-55$ atom $(0.5-1.5 \mathrm{~nm})$ cobalt nanoparticles and preferred chemisorption sites depend on the cluster structure (whether it is fcc or icosahedral), size and surface coverage. In addition, we found a strong influence of $\mathrm{CO}$ on the magnetism of the cluster, leading to magnetic moments smaller than in the bulk, at variance with pristine clusters which have magnetic moments larger than the bulk. Our findings point at important changes in catalytic properties of Cobalt at the nanoscale. Our theory suggests that at the nano-scale cluster size and surface coverage control the catalysis of $\mathrm{CO}$ through the FisherTropsch synthesis.

\section{Calculations of plasmons in metallic nanoparticles used for SERS}

We have calculated and measured the opto-electronic properties of gold metallic nano-spheres. We have characterized the chemical composition and electronic structure of these gold nanospheres using x-ray absorption measurements at the ALS at LBL. We calculated the surface nano-plasmons of the spheres to design nanoparticles whose structures are optimized to enhance Raman scattering in Surface Enhanced Raman Scattering (SERS) experiments. This work was performed in collaboration with Chad Talley (CMS), Steve Lane (PAT) and Tomas Huser (CMS/UC Davis) who are fabricating metallic nano-spheres for applications in biodetection using SERS signals.

\section{Synthesis and Characterization of Silicon and Germanium Nanoparticles Using Physical Vapor Deposition}

One of the most important technical challenges of this Si was to develop the ability to produce nanoparticles with well controlled size, shape, composition, and surface structure. Using our Physical Vapor Deposition (PVD) technique we have developed techniques to reproducibly fabricate silicon and germanium nanoparticles with narrow size distributions and doped with atoms which can be used for labeling.

We have focused our PVD work on changing the surface chemistry of the gas phase produced silicon nanoparticles for further chemical modification. To do so, a new procedure has been developed in which the nanoparticles surface is terminated with methanol after growth in the UHV chamber. Methanol molecules are chosen because they have been previously shown to bond in a controlled manner on silicon surfaces. The methanol terminated silicon nanoparticles show a clear sub-oxide feature in silicon $2 \mathrm{p}$ photoelectron spectra indicating a covalent attachment of the methanol molecules to the nanoparticle surface. No such feature is seen the $2 p$ photoelectron spectra from the unpassivated silicon nanoparticles. From the photoemission data we estimate the methanol surface termination to be one monolayer. We believe the attachment process works via a break-up of the OH-group and subsequent formation of Si-O-R bonds. Preliminary $\mathrm{x}$-ray absorption experiments show that the methanol terminated silicon nanoparticles are air stable for at least 24 hours with no noticeable change in the electronic 
structure. Previously we were not able to remove the silicon nanoparticle from the UHV system without significant oxidation. Surprisingly, the absorption edge shift of the unpassivated and methanol terminated nanoparticles is the same when compared to bulk silicon. We would expect that changes in the surface passivant configuration to have a significant impact on the nanoparticle electronic structure however more samples need to be measured to determine the degree of this impact. This research allows us to remove the silicon nanoparticle from the UHV system for characterization by other techniques and allows for further modification of the nanoparticle surface chemistry.

We have also developed a procedure for doping germanium nanoparticles with erbium. These nanoparticles are being used in NHI for remote sensing applications.

\section{Chemical Synthesis and Characterization of Nanoparticles and Superlattices and Wires}

In addition to Physical Vapor Deposition synthesis, we have also made significant progress in the chemical synthesis and characterization of $\mathrm{Si}, \mathrm{Ge}, \mathrm{CdSe}, \mathrm{Fe}_{2} \mathrm{O}_{3}$, and $\mathrm{InP}$ nanoparticles. We have demonstrated the first growth and optical characterization of $\mathrm{CdSe}$ and $\mathrm{Fe}_{2} \mathrm{O}_{3}$ faceted nanoparticle superlattices. As these superlattices grow, they self organize and size select so that the size distribution of the nanoparticles is much smaller than the size distribution in the original solution (see Fig.12). This reduction in size distribution in combination with the uniform spacing between the nanoparticles in the superlattice gives the superlattices unique optical properties, such as extremely narrow emission line widths.

We have developed a method for growing highly crystalline Ge nanowires based on the high temperature, high pressure decomposition of organometallic precursors to work in conventional glassware. Unlike conventional Vapor-Liquid-Solid (VLS) growth techniques, which require catalytic seeds mounted on a substrate, this approach does not require any catalytic seeds. Therefore, we were able to perform the first investigations of the growth properties, such as growth direction, of the nanowires in the absence of the influence of the substrate. Our wires are also free of impurities that might be introduced by the substrate or seed particles. Our initial experiments with InP have shown that this method developed for the synthesis of germanium nanowires can also be applied to the syntheses of different one-dimensional nanostructures.

We have also developed new precursors and solvents to obtain 2-5 nm isometric germanium particles soluble in traditional solvents like toluene and chloroform. Silicon nanoparticles with sizes ranging from $10 \mathrm{~nm}$ to as small as $1 \mathrm{~nm}$ have been obtained by the use of both highpressure and vacuum techniques. Characterization of these nanoparticles with electron

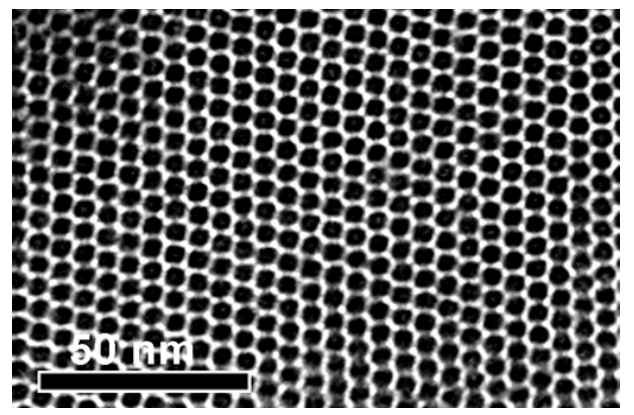

Fig.12 A CdSe Qdot superlatttice microscopy showed high crystallinity and extreme stability of the nanoparticles to oxidation.

In the final phase of the SI we focused on synthesizing faceted superlattices from our nanoparticles. We successfully fabricated three-dimensional superlattices, of $\mathrm{CdSe}$ and $\mathrm{Fe}_{2} \mathrm{O}_{3}$ nanoparticles. These superlattices were grown to microscopic sizes, which made possible, for the first time, direct characterization of their optical properties. Our experiments (see Fig. 12) show that these unique structures have extremely narrow fluorescence 
lines and a high quantum yield determined by the uniform size of the constituent nanoparticles.

\section{Synthesis and Applications of Functionalized CdSe Nanoparticles}

We have synthesized and characterized the properties of fluorescent CdSe nanoparticles (Qdots) for two types of biological applications that are likely to emerge in the next few years and may position LLNL as a central player in nano-biotechnology. These are; (i) the detection of signaling pathways in live organisms which has potential for early detection of viral attacks, and (ii) developing dual probes for invasive and non-invasive detection which has potential for tracking labeled organisms such as stem cells. We have focused on synthesizing these probes, or adapting existing syntheses for a range of nano-biotechnology applications, including:

1. We have collaborated with Fanqing Chen (LBNL and Comparative Cancer Center-UCSF) studying the labeling of live cells and using nanoparticles to target their nuclei. A joint patent (LLNL/LBNL) has been submitted by both laboratories. This work has attracted worldwide recognition

2. We collaborated with Julie Herberg (Center for National Security Applications of Magnetic Resonance-CMS, LLNL) and Fanqing Chen to use of fluorescent nanoparticles as probes for magnetic resonance imaging (MRI-Qdots). We developed a probe that can be detected by invasive (fluorescence) and non-invasive (magnetic resonance) means. A patent disclosure covering the synthesis of the MRI-fluorescent nanoprobes has been submitted.

\section{Applications of carbon nanotubes as novel nanoscale stationary phases for molecular detection and separation}

We have investigated the potential of carbon nanotubes to be used as a novel, nanoscale stationary phase for molecular separation. Carbon nanotube arrays offer ease of fabrication, mechanical and chemical robustness and a phenomenally high surface to volume ratio. These properties hint at the potential of carbon nanotube arrays to serve as a basic platform for building separation elements for micro- and nano-fluidic systems. However, to realize this potential we need to understand how chemical functionalities interact with bare and functionalized carbon nanotube surfaces. We pursued a strategy based on three complementary approaches: (1) Molecular simulations of interactions of functional groups with carbon nanotubes; (2) Direct measurements of the strength of the molecular interactions with carbon nanotube sidewalls using Chemical Force

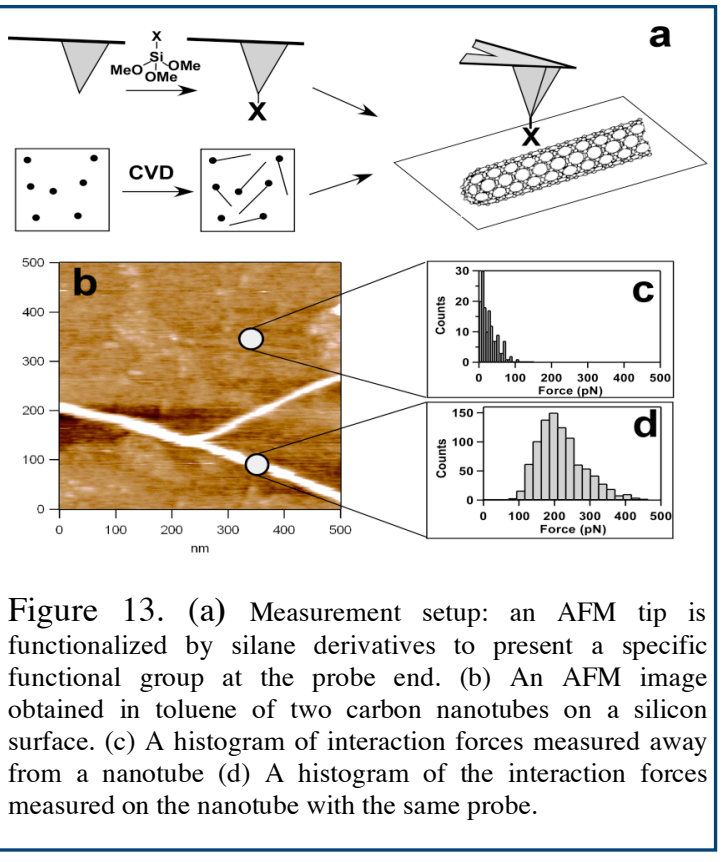
Microscopy, and (3) Direct testing of carbon nanotube separation capacity in conventional and microfabricated chromatography columns. 


\section{Chemical Force Microscopy Measurements separation}

We have functionalized sharp AFM tips with different chemical functionalities and then measured the binding force or the interactions of the tip with the sidewall of pristine clean single wall carbon nanotubes in toluene (Fig. 13a). Control experiments shown on the Fig. 4b demonstrate that our instrument is capable of measuring adhesion forces in a specific location and that the interactions of a functionalized AFM tip with carbon nanotube sidewall are clearly distinct from the interaction of the tip with the background silicon oxide surface. Significantly, our calculations of a tip-nanotube contact area for our experiments showed that on average we are measuring interactions of a single functional group with the nanotube sidewall.

To validate the simulation results we have used a model developed by Dudko and Urbakh which predicts a distribution of the binding forces based on the Morse potential parameters of the interactions. We have used this model and our calculated interaction potentials to generate the predicted binding force distributions for interactions of three different functional groups with the nanotube sidewalls. A comparison of the calculated distributions with the experimental results shows extremely good agreement with both the peak position and the shape of the distributions for all functional groups that were tested (Fig. 13). The results presented in Fig. 13 are even more remarkable if we consider that all three curves were generated using only one fitting parameter- the damping constant $\gamma$ for the molecule-nanotube interactions (and all 3 values of $\gamma$ fall within one order of magnitude of one another.). These results are valuable for several reasons:

This is the first direct measurement of the interaction strength of a single functional group. All previous measurements involved ensembles of chemical functionalities. They represent the first direct experimental comparison of measured interaction strength in a nanoscale system with the simulations results. They represent a direct measurement of the functional group interactions with carbon nanotube sidewalls.

These experiments provide a tool for directly evaluating the affinities of functional groups to nanotube sidewalls. The final phase in this project is to transfer this information into actual nanotube-based separation systems. We have pursued two approaches for this stage: We have tested nanotube separation capabilities in conventional columns packed with carbon nanotubes, and we have developed fabrication protocols for micro-

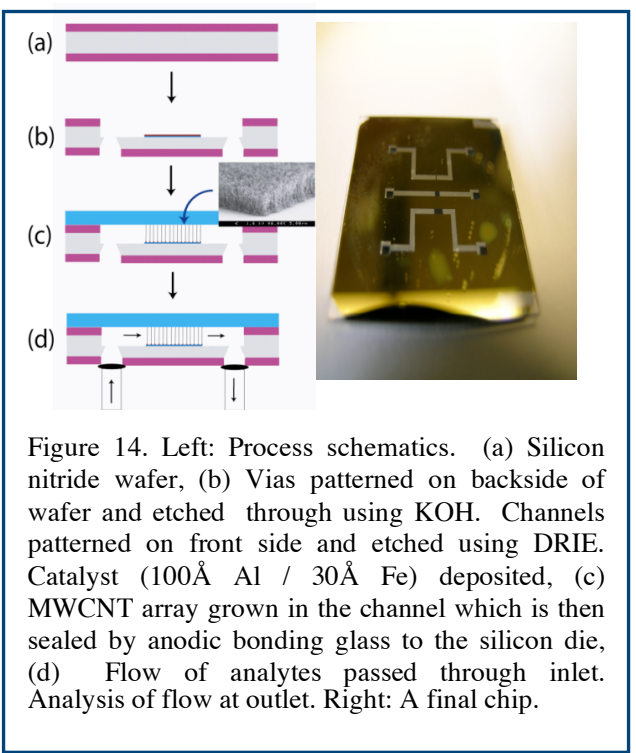
columns that contain free-standing nanotube arrays as a stationary phase.

\section{Carbon Nanotube Device Fabrication and Characterization}

We have examined multi-wall carbon nanotubes as a separation medium by packing standard $150 \mu \mathrm{m}$ capillaries and flowing through an analyte. Preliminary results indicate that the nanotubes appear to be a reasonable separation medium. For example, maltohexose molecules are retained by the CNT matrix and then released. We have also succeeded in fabricating silicon-based chips that contained nanotube beds that span the whole cross-section of a sealed microfluidic channel (Fig. 14). 
Final Report: 03-SI-001, PI: A.J. Williamson 
Final Report: 03-SI-001, PI: A.J. Williamson

\section{PUBLICATIONS, PATENTS, INVITED TALKS AND OTHER SI RELATED ACTIVITIES}

\section{Publications:}

A. Puzder, A.J. Williamson and G. Galli, Self-Healing of CdSe Nanoparticles: A First Principles Study, Phys. Rev. Lett. 92, 217401 (2004). UCRL-JRNL-207529

A. Puzder, A.J. Williamson, N. Zaitseva, G. Galli, L. Manna and A.P. Alivisatos The Effect of Organic Ligand Binding on the Growth CdSe Nanoparticles Probed by Ab-Initio Calculations, Nano Letters 4, 2361 (2004). UCRL-JRNL-207520

A.J. Williamson, C. Bostedt, T. van Buuren, T. M. Willey, L. J. Terminello, and G. Galli, "Probing the Electronic Density of States of Germanium Nanoparticles: A Method for Determining Atomic Structure," Nano Letters, 2004, 4(6) 1041-1045. UCRL-JRNL-203560

A.J. Williamson, F.A. Reboredo and G. Galli, Chemisorption on semiconductor nanocomposites: A mechanism for hydrogen storage, Appl. Phys. Lett. 14, 2917 (2004). UCRL-JRNL-203848

C. Bostedt, T. van Buuren, T. M. Willey, A. J. Nelson, N. Franco, T. Möller, L. J. Terminello, "Photoemission Spectroscopy of Germanium Nanocrystal Films," Journal of Electron Spectroscopy and Related Phenomena, 2002, 126(1-3), 117-124.UCRL-LR-148228N. UCRL-JC-149020

C. Bostedt, T. van Buuren, C. Heske, N. Franco, T. M. Willey, T. Möller, and L. J. Terminello, Strong confinement in the conduction band edge of germanium nanoparticles. Appl. Phys. Lett. 84, 4056 (2004). UCRL-JC-150516

C. Bostedt, T. Van Buuren, J. Plitzko, T. Moller, L.J. Terminello, Controlled gas-phase synthesis of germanium nanocrystal films, J. Phys. Condens. Matter. Vol 15, p1017 (2003).

C. Bostedt, T. van Buuren, T. M. Willey, A. J. Nelson, N. Franco, T. Möller, L. J. Terminello, "Characterization of Ge-nanocrystal films with Photoelectron Spectroscopy," Nuclear Instruments and Methods in Physics Research B, 2003, 199, 402-405.

C. Bostedt, T. Van Buuren, T. M. Willey, and L. J. Terminello, "Controlling the Electronic Structure of Nanocrystal Assemblies by Variation of the Particle-Particle Interaction," Applied Physics Letters, 2004, 85(22), 5334-5536. UCRL-JRNL-206909

C. Bostedt, T. van Buuren, T. M. Willey, and L. J. Terminello, Controlling the electronic structure of nanocrystal assemblies by variation of the particle - particle interaction, Appl. Phys. Lett. 85, 5334 (2004).

C. Bostedt, T. van Buuren, T. M. Willey, N. Franco, L. J. Terminello, C. Heske, and T. Möller, "Strong Quantum Confinement Effects in the Conduction Band of Germanium Nanocrystals," Applied Physics Letters, 2004, 84(20), 4056-4058. UCRL-JRNL-203356

C. Bostedt, T. Van Buuren, T.M. Willey, A.J. Nelson, N. Franco, T. Moller, L.J. Terminello Charaterization of Germanium Nanocluster Films with photoelectron spectroscopy, Nucl. Instr. and Meth. Vol.199, p402 (2003). UCRL-JC-138031

D. Gerion, Fluorescence Imaging in Biology using Nanoprobes. In "Nanotechnologies for the Life Sciences: Nanosystem Characterization tools for nanoystems in the Life Sciences", Edited by Challa S.S.R. Kumar, WileyVCH Verlag GmbH \& Co, Weinheim, In Press. UCRL-JRNL-205304

D. Gerion, N. Zaitseva, C. Saw, M.F. Casula, S. Fakra, T. van Buuren, G. Galli, Solution Synthesis of Germanium Nanocrystals: Success and Open Challenges, Nano. Lett. 4 (4): 597-602 (2004).

D. Gerion, Prospects for studying live cells using semiconductor quantum dots. Proceedings SPIE, 5704, 77-85, (2005). UCRL-JRNL-201498

F. Chen and D. Gerion, Fluorescent CdSe/ZnS Nanocrystal-Peptide Conjugates for Long-term Imaging and Nuclear Targeting in Living Cells. Nano Lett. 4, 1827-1832, (2004). UCRL-JRNL-205304 
Final Report: 03-SI-001, PI: A.J. Williamson

F. Reboredo and A.J. Williamson, Optimized nonorthogonal localized orbitals for linear scaling quantum Monte Carlo calculations, Physical Review B, Rapid Communications 71, 121105 (2005). UCRL-JRNL-205574

F.A. Reboredo and G. Galli, Theory of alkyl-terminated silicon quantum dots, Journal of Physical Chemistry B 109, $1072(2005)$.

Here is the list of papers published with the SI finding from our group (including 1paper still in review):

J.Y. Raty, G. Galli, C. Bostedt, T. Van Buuren, L.J. Terminello, Quantum Confinement and Fullerene like Surface Reconstruction in Nanodiamonds, Phys. Rev. Lett. Vol. 90, p401 (2003). UCRL-JC-148729

J.Z. Zhang, A.M. Schwartzberg, T Norman, CD Grant, J. Lui, F. Bridges, T. van Buuren, Comment on "gold nanoshells improve single nanoparticle molecular sensors" Nano Lett., 5, 809, (2005).

Luca Dal Negro, Sebastien Hamel, Natalia Zaitseva, Jae Hyung Yi, Andrew Williamson, Mike Stolfi, Jurgen Michel, Giulia Galli, and Lionel C. Kimerli, Synthesis, Characterization and Modeling of Colloidal and Thin Film Silicon Nanocrystals, IEEE J.Sel. Top. Quant. Electr., 2006, V. 12 (6), Part 1, 1151 - 1163. UCRL-JRNL-216732

M. C. LeMieux, R. W. Friddle, G. Cicero, A. B. Artyukhin, V. V. Tsukruk, J. C. Grossman, G. Galli, A. Noy Single Functional Group Interactions with Carbon Nanotube Sidewalls, Nature Nanotechnology, in review (2007) UCRL-JRNL-220263

M. Stadermann, A. D. McBrady, B. A. Dick, V. Reid, A. Noy, R. E. Synovec, O. Bakajin, Ultra-fast gas chromatography on single-wall carbon nanotube stationary phases in microfabricated channels, Analytical Chemistry, v. 78, p. 5639-5644, (2006), UCRL-JRNL-219049

N. Drummond, A.J. Williamson, R.J. Needs and Giulia Galli, Electron emission from diamondoids: A diffusion quantum Monte Carlo study, Physical Review Letters 95, 096801 (2005). UCRL-JRNL-209819

N. Zaitseva, J. Harper, D. Gerion and C.K. Saw, Unseeded growth of germanium nanowires by Vapor-Liquid-Solid mechanism. App. Phys. Lett., 86, 053105-1-3, (2005). UCRL-JRNL-20149

Natalia Zaitseva, Liberato Manna, Daniele Gerion, and Cheng K. Saw, Precipitation of Selenium from CdSe Nanocrystal Solutions, Adv. Mater. 17 1321, (2005). UCRL-JRNL-203438

Natalia Zaitseva, Zu Rong Dai, Francisco R. Leon, and Denise Krol, Optical Properties of CdSe Superlattices, J. Am. Chem. Soc. 127 10221, (2005). UCRL-JRNL-203438

R. W. Meulenberg, T. van Buuren, K. M. Hanif, T. M. Willey, G. F. Strouse, L. J. Terminello, "Structure and Composition of Cu-Doped CdSe Nanocrystals Using Soft X-ray Absorption Spectroscopy," Nano Letters, 2004, 4(11) 2277-2285. UCRL-JRNL-204584

Robert W. Meulenberg, Tony van Buuren, Khalid M. Hanif, Trevor M. Willey, Geoffrey F. Strouse, Louis J. Terminello, Structure and Composition of $\mathrm{Cu}$ Doped CdSe Nanocrystals Using Soft X-ray Absorption Spectroscopy, Nano. Lett. 4, 2277 (2004).

S.-C. Huang, A.B. Artyukhin, Y. Wang, J.-W. Ju, P. Stroeve, A. Noy Persistence Length Control of the Polyelectrolyte Layer-by-Layer Self-Assembly on Carbon Nanotubes J. Am. Chem. Soc., v. 127 (41), p.1417614177 (2005), UCRL-JRNL-214130

T. J. Norman, Jr., C. D. Grant, D. Magana, J. Z. Zhang, J. Liu, D. Cao, F. Bridges, T. Van Buuren, Near Infrared Optical Absorption of Gold Nanoparticle Aggregates, J. Phys. Chem. B, Vol 106, p 7005 (2002).

T. M. Willey, C. Bostedt, T. van Buuren, J. E. Dahl, S. G. Liu, R. M. K. Carlson, R. W. Meulenberg, E. Nelson, and L. J. Terminello, "Observation of Quantum Confinement in the Occupied States of Diamond Clusters," Physical Review B, 2006, 74 ,205432. UCRL-JRNL-221748

T. M. Willey, C. Bostedt, T. van Buuren, J. E. Dahl, S. G. Liu, R. M. K. Carlson, L. J. Terminello, and T. Möller, "Molecular Limits to the Quantum Confinement Model in Diamond Clusters," Physical Review Letters, 2005, 95, 113401 UCRL-JRNL-211271

T. M. Willey, C. Bostedt, T. van Buuren, J. E. Dahl, S. G. Liu, R. M. K. Carlson, L. J. Terminello, T. Moller, Molecular Limits to the Quantum Confinement Model in Diamond Clusters, Phys. Rev. Lett, 95 113401, (2005). UCRL-JRNL-221748 
Final Report: 03-SI-001, PI: A.J. Williamson

T. van Buuren, C. Bostedt, B.R. Taylor, T. Willey, L. Hope-Weeks, B. Weeks, L.J Terminello, X-ray Absorption Study of Colloidal Ge Quantum Dots, Proceedings of SPIE Volume: 4807, p 1 (2002). UCRL-JC-151034

Trevor M. Willey, Andrew L. Vance, T. van Buuren, C. Bostedt, L. J. Terminello, C. S. Fadley, Rapid Degradation of Alkanethiol-Based Self-Assembled Monolayers on Gold in Ambient Laboratory Conditions, Surface Science, 2005, 576 (1-3), 188-196. UCRL-JRNL-220642

Y.-H. Kim, Y. Zhao, A.J. Williamson and S. Zhang, Non-dissociative adsorption of $\mathrm{H}_{2}$ molecules in light-elementdoped fullerenes, Physical Review Letters. (2006) UCRL-JRNL-209820

Zaitseva, L. Manna, D. Gerion and C.K. Saw, Faceted Crystals Precipitated from CdSe Nanocrystal Solutions: Superlattices or Bulk Selenium? Advanced Materials, 17, 1321-1324, (2005). UCRL-JRNL-203438

\section{Patent Applications}

O. Bakajin, A. Noy, Microfluidic Sieve Using Intertwined Free-Standing Carbon Nanotube Mesh as Active Medium, US application 10/613,960

F. Chen and D. Gerion, Fluorescent Semiconductor Nanocrystal-Peptide Conjugates for Long-term, Nontoxic Imaging and Nuclear Targeting in Living Cells. Patent US Serial \# 60/607286.

Disclosure of Record of invention: F. Chen, D. Gerion, J.W. Gray, T.J. Lowery, D.E. Wemmer, A. Pines, Xenon nanocage-coated quantum dots as a multi-modality imaging agent.

O. Bakajin and A. Noy, Microfabricated Channel with Patterned Carbon Nanotube Array Active Element and a Fabrication Strategy for Such Device, DOE Invention Case No: S-99,

O. Bakajin and A. Noy, The Use of Free Standing Carbon Nanotubes Arrays as Separation and Concentration Medium, DOE Invention Case No: S-99, 687

\section{Invited Talks}

F. Reboredo, Dept of Applied Mathematics, University of Western Ontario, London Ontario, 2005.

F. Reboredo, Dept of Physics, Vanderbilt University Nashville TN, 2005.

G. Galli, Pacifichem, Waikiki, Hawaii, December 15-20, 2005, Waikiki

G. Galli, Materials Research Society, Boston, MA, December 2005.

G. Galli, Princeton Materials Research Colloquium, Nov.14, 2005

G. Galli, MIT Materials Science Colloquium, November 4, 2005.

G. Galli, Psi-K General European Electronic Structure and Materials Science Conference Plenary Speaker, Germany, September 17-21, 2005.

G. Galli, Car-Parrinello Molecular Dynamics symposium, Ascona, Switzerland, Sept. 2-8, 2005.

G. Galli, DOE SciDac 2005, Plenary Speaker, San Francisco, June 26-30, 2005.

G. Galli, American Chemical Society, Division of Physical Chemistry, San Diego, CA, March 2005.

G. Galli, March Meeting of the American Physical Society, Los Angeles, CA, March 2005.

G. Galli, "Condensed Matter Physics Seminar", Sandia National Laboratory, Albuquerque, NM, February 2005.

G. Galli, "Physical Chemistry Seminar", UCLA, Los Angeles, CA, December 6, 2004.

G. Galli, International Society for Optical Engineering, Philadelphia, PA, October 25-28, 2004.

D. Gerion, MRS, Fall meeting, Boston, Nov. 2005

D. Gerion, ACS, spring meeting, San Diego, CA - March 2005 
Final Report: 03-SI-001, PI: A.J. Williamson

D. Gerion, SPIE, The international Society for Optical Imaging, 2005.

T. van Buuren, Pacifichem, Waikiki, Hawaii, December 15-20, 2005, Waikiki

T. van Buuren "Power of soft X-ray emission spectroscopy in the study of semiconductor nanoparticles" Analytical Chemistry Section, Soft x-ray emission spectroscopy, Pacifichem 2005.

T. van Buuren "Soft x-ray emission study of silicon nanomaterials" Workshop on photon-in photon-out x-ray spectroscopy in materials science, Advanced Light Source user meeting Berkeley 2005.

T. van Buuren "Manipulation and Quantitative Interrogation of Nanostructures" UC Merced, Molecular Science Seminar, Nov. 2005.

T. van Buuren "Manipulation and Quantitative Interrogation of Nanostructures" Science highlights seminar at Steacie Institute for Molecular Sciences, National Research Council, Canada 2005.

T. van Buuren "Nanostructure properties characterized using soft x-ray spectroscopy" 229th ACS National Meeting San Diego 2005.

T. van Buuren "Influence of the strain, surface structure and particle-particle interaction on the occupied and empty density of states in group-IV nanostructures" Symposium on group IV semiconductor nanoparticles, MRS meeting Boston 2004.

T. van Buuren "Soft x-ray emission and fluorescence study of nanomaterials" Workshop on photon-in photon-out x-ray spectroscopy in materials science, Advanced Light Source user meeting Berkeley 2004.

T. van Buuren "X-ray absorption and emission studies of semiconductor nanoparticles" 14th International Conference on Vacuum Ultraviolet Radiation Physics Cairns Australia 2004 (upgraded to invited talk at conference)

T. van Buuren "Probing the Physical and Electronic Properties of Nanomaterials" 53 RD Annual Denver X-ray Conference, Steamboat Springs Colorado 2004.

T. van Buuren "Electronic properties of semiconductor quantum dots" ALS/CXRO seminar series Berkeley 2004.

T. van Buuren, "Electronic and Surface Structure of Semiconductor Quantum Dots" American Physical Society National Meeting, Austin Texas March 3-9 2003.

T. van Buuren, "Novel Applications of Synchrotron Radiation in the Study of Nano-scale Materials" Workshop on Materials Science with Synchrotron Radiation, CLS users meeting Saskatoon Saskatchewan, Nov. 152002.

T. van Buuren, " Particle size and surface termination effects on the band edges of semiconductor nanoparticles "International symposium on Optical Science and Technology, SPIE 47th Annual Meeting Seattle, July 7-11 2002.

. van Buuren, "Synchrotron radiation measurements of the electronic structure in semiconductor nanoparticles". UCSD Department of Chemistry Inorganic Seminar, June 5 2002.T. van Buuren, $229^{\text {th }}$ ACS National Meeting San Diego 2005.

T. Wiley, 11th International Conference on New Diamond Science and Technology (ICNDST) and 9th Applied Diamond Conf. (ADC), North Carolina, May 16, 2006.

A. Williamson, Pacifichem, Waikiki, Hawaii, December 15-20, 2005, Waikiki

A. Williamson, General Colloquium at California State University Northridge, Fall 2005

A. Williamson, University of California at Davis, Department of Applied Science, 2005.

A. Williamson, Materials Research Society, Boston, 2004.

A. Williamson, Santa Clara University, 2004.

A. Williamson, School of Engineering, UCLA, 2004. 
Final Report: 03-SI-001, PI: A.J. Williamson

\section{SI Related Activities}

C. Bostedt, PhD Thesis "Electronic Structure of Germanium Nanocrystal Films Probed with Synchrotron Radiation," May 1, 2002

G. Galli, American Physical Society, Division of Computational Physics, Chair-Elect (February 2005).

G. Galli, Member of Editorial Board of SIAM "Multiscale Modeling and Simulation" Journal (1/1/05-12/31/07).

G. Galli, Resource Specialist to the Task Force on Nanotechnology for Congressman Honda (CA) (2004).

G. Galli, Member of Executive Committee of American Physical Society, California Section (December 2004).

G. Galli, Member of Board of Experts of the Italian Ministry of Education, University and Scientific Research.

G. Galli, Member of the Molecular Foundry (University of California at Berkeley) Scientific Advisory Board.

G. Galli, Member of the Scientific Advisory Board for the MIT Institute for Soldier Nanotechnologies.

G. Galli, Member of Advisory Board of the "International Workshop on computational material science, total energy and force methods" (bi-annual workshop in its $12^{\text {th }}$ year).

G. Galli, Organizer of Symposium "Computational crystallography applied to extreme conditions" on behalf of IUCr commission on high-pressure, Florence, Italy, August 2005.

G. Galli, Chairperson of the "International Workshop on computational material science, total energy and force methods", Trieste, Italy, 2005.

T. van Buuren, Steacie Institute for Molecular Sciences NRC Ottawa Canada June 2005

A. Williamson, Director of Computational Chemistry and Materials Science Summer Institute, LLNL 2005.

A. Williamson, Director of Computational Chemistry and Materials Science Summer Institute, LLNL 2006

A. Williamson, Organizer of DCOMP Nanomaterials simulation session at 2005 APS Meeting. 